\title{
SEMBLANZAS
}




\section{EL MERTON ESENCIAL *}

\section{Gonzalo Cataño **}

La ya legendaria serie de la Universidad de Chicago, "The Heritage of Sociology", dedicada a difundir los clásicos de la ciencia social norteamericana y europea, ha consagrado su última entrega a Robert K. Merton. El volumen contiene el Merton esencial, el Merton imprescindible, el que todos debemos consultar para adquirir una comprensión básica de sus contribuciones a la sociología del siglo XX. Con destreza y amplio conocimiento de la obra del renombrado sociólogo estadounidense, el editor -el polaco Piotr Sztompka - ofrece a los lectores una muestra de los textos más representativos del autor de Teoría y estructura sociales. El interesado podrá encontrar aquí el ensayo clásico sobre la anomia y la conducta desviada; el examen de las funciones latentes y manifiestas; la discusión de las teorías de alcance medio y la codificación en sociología (los paradigmas), ilustrados con los casos del análisis funcional, el análisis estructural y el estudio social de las ideas (el paradigma de la sociología del conocimiento). A ello se suma una presentación de la ciencia como institución social y una selección del elegante, persuasivo y útil vocabulario mertoniano dirigido a desentrañar los aspectos menos evidentes de la dinámica social: la estructura de oportunidades, la ambivalencia sociológica, el conjunto de roles, el efecto Mateo, la ignorancia especificada, las duraciones socialmente esperadas y la profecía que se cumple a sí misma.

Merton ha enriquecido el léxico de las ciencias sociales con una arquitectura conceptual que ha llevado a precisar tanto la teoría general como el trabajo en algunos campos especiales de la sociología ${ }^{211}$. Para aludir a los menos conocidos por la audiencia española, mencionaremos la noción de "ignorancia especificada", el útil predicado metodológico que demanda no sólo manifestar el vacío en un campo determinado del conocimiento, sino la exigencia de hacerlo explícito mediante su exacta delimitación. En el

\footnotetext{
*A propósito de Robert K. Merton. On Social Structure and Science, edición a cargo de Piotr Sztompka (Chicago: The University of Chicago Press. 1996). serie Heritage of Sociologv".

Sociólogo. Profesor titular de la Universidad Pedagógica Nacional. Autor de La Sociología en Colombia (tercera edición, 1997). Educación y estructura, social (1989) y La artesanía intelectual (1995).

211 Buena parte del impacto de Merton en la investigación empírica se debe al rigor de su elaboración teórica, al valor operativo de SUS conceptos y a su utilidad para orientar la observación de los hechos. EI vocabulario mertoniano, un glosario no inferior a medio centenar de términos, compuesto de ideas, conceptos y protoconceptos y este ultimo es ya un ejemplar de su ondulante $y$ sutil repertorio-, ha sido empleado en los más diversos campos del análisis social. Algunos de estos vocablos son de su propia cosecha y otros han sido tomados de los clásicos o de los analistas contemporáneos, pero siempre con desarrollos propios que amplían su contenido y alcance. El concepto de "grupo de referencia, por ejemplo, pertenece al sociólogo y psicólogo social Herbert Imán, pero en manos de Merton adquirió una extensión y riqueza inesperadas. Lo mismo ocurrió con la "anomia" de Durkheim, con la noción de "función" difundida por la antropología social inglesa o con la "definición de una situación" de William I. Thomas, concepto que ha formalizado hasta alcanzar en sus escritos la categoría de "teorema".

Un trabajo interesante, y urgente para un adecuado manejo de las contribuciones teóricas de Merton, es la organización y sistematización -acompañada de las necesarias ejemplificaciones- de su variado repertorio. Esta tarea sólo podría realizarla alguien cercano al autor y lo suficientemente familiarizado con su vasta, ensayística y dispersa obra publicada en periódicos, boletines, revistas, simposios y libros colectivos. Su producto sería de gran ayuda tanto para los investigadores activos como para el mismo Merton. Aquel glosario disminuiría los peligros de la "obliteración por incorporación", las consecuencias de la irónica y común operación de olvidarse de la fuente de una contribución a medida que se propaga su uso. Cuando una idea, un vocablo o un concepto pasa al repertorio común, surge la tendencia a la obliteración de su origen ¿Quién recuerda que la extendida y muy querida noción de "altruismo" se debe al vituperado creador del positivismo Auguste Comte? Algo parecido está ocurriendo con "la profecía que se cumple a sí misma", el popular concepto engendrado por Merton en los años cuarenta.
} 
estado actual de la ciencia social no es suficiente confesar la ignorancia; ello es sólo el primer paso para superar los obstáculos más apremiantes. El siguiente y de mayor valor estratégico, es reconocer expresamente lo que no se sabe pero que debe ser conocido para remover las dificultades que impiden el avance del conocimiento. Algo parecido ocurre con la evasiva noción de "duración socialmente esperada", dirigida a capturar los efectos de las definiciones del tiempo desarrolladas por los actores. Como se sabe, el tiempo social y el tiempo real, el de los relojes y calendarios, no siempre son los mismos. La duración esperada de residencia en un barrio tiene consecuencias en el grado de integración de sus moradores con el entorno. Si se elige el lugar como una etapa transitoria, las relaciones con el vecindario serán pobres y ligeras a pesar de que se viva allí algunos años, frente a aquellos que consideran el barrio como el emplazamiento de su vida o de una parte significativa de ella.

Pero el concepto central que enlaza y confiere sentido a los ensayos de On Social Structure and Science, y por extensión a toda la obra de Merton, es la noción de estructura y más exactamente de "estructura de oportunidad". Este permanente interés proviene de su enfoque de la sociología, disciplina que tiende a definir como la ciencia que trata de descubrir la manera en que las actitudes y destino de los hombres y mujeres se ven influidos, aunque no determinados en todos sus detalles, por su posición en ciertos tipos cambiantes de estructuras sociales y culturales. Si esto es así, no es extraño encontrar que todo el proyecto intelectual de Merton, como el de su mentor a distancia el francés Émile Durkheim, haya girado alrededor del contenido de la idea de estructura. Esta eterna jornada analítica -donde la descripción es sólo el terreno de la ejemplificación-, lo ha llevado a transitar los más diversos campos del análisis social con resultados positivos para el desarrollo de la sociología del conocimiento, de las comunicaciones, de las profesiones, del tiempo y de la ciencia, especialidad esta última de la cual es su fundador y primer sistematizador.

Si bien para Merton las estructuras son sendas que prescriben la conducta de los actores—que la organizan en una u otra dirección hasta hacerla repetitiva y estable-, ellas no son ajenas a las contradicciones. La norma que fija un camino y señala su destino, puede estar negada o neutralizada por otra que sugiere un rumbo contrario. $A$ esto denornina "ambivalencia sociológica", es decir, las tendencias normativas opuestas, las normas y contranormas, que orientan el desempeño de un rol. De un médico se espera que tenga una relación neutral y objetiva con sus enfermos, pero también se le exige que muestre interés y afecto por el padecimiento de sus dolientes. Este enfoque evita la rigidez de la mayoría de las nociones de estructura en uso, y al final da vida a la libertad y al juego de los actores en el escenario social.

La fluidez de esta perspectiva se afinca en la teoría del rol, un marco de referencia muy extendido en la sociología norteamericana y nada popular en los medios latinoamericanos. El bordado analítico de Merton no se desprende de las clases sociales o de las relaciones que establecen los individuos en el proceso de producción, dos abstracciones macro que tienden a obscurecer los desarrollos micro hasta convertirlos en esquematizaciones vacías. Su fundamento son las conductas pautadas que se espera de las personas que ocupan determinadas posiciones. Aquellas pautas no son en ningún momento únicas y estables, están por el contrario sujetas a tensiones según las demandas de la variedad de posiciones ocupadas por un individuo. Un estudiante tiene relaciones de camaradería con sus compañeros de salón, pero cuando trabaja algunas horas como funcionario de la biblioteca debe orientarse mediante normas que tienden a cancelar los lazos de intimidad y afecto. Ahora sus amigos son meros usuarios de un servicio que él debe atender sin consideraciones personales. Esta tensión da lugar a 
conflicto de roles y en algunos casos a verdaderos sufrimientos a los conocidos embrollos, confusiones y angustias que acompañan lo que regularmente se conoce como la vida cotidiana.

La mirada de Merton está por lo tanto dirigida a estudiar al individuo anclado en una tupida red de relaciones sociales no exentas de conflictos y aun de severas contradicciones. Algo similar ocurre con la esfera cultural, con ese conjunto de valores que nutren la conducta habitual de los individuos de una sociedad o de un grupo determinado. Aquí el acento está puesto en las consecuencias de las ideas y de las creencias compartidas. Hay objetivos comunes, pero los caminos para lograrlos son diversos y a veces encontrados. Los grupos implicados en el tráfico de drogas no son enemigos del statu quo, no desean subvertir el orden social; por el contrario, mediante vías negativamente sancionadas quieren alcanzar un valor muy estimado en la sociedad: el éxito económico. Aquí surge con toda su fuerza la estructura de oportunidad, "la escala y distribución de condiciones que proveen varias probabilidades para los individuos y los grupos de alcanzar sus objetivos". Los actores de Merton nunca están quietos; se mueven en varias direcciones, y ante las presiones estructurales buscan los más diversos caminos para alcanzar sus fines.

Como lo anuncia el título, On Social Structure and Science, incluye también varios trabajos de sociología de la ciencia, una de las especialidades más activas de la sociología contemporánea que ha crecido al lado del florecimiento de la filosofía y la historia de la ciencia. Para Merton la ciencia es una institución social integrada por un grupo de individuos comprometidos con el avance del conocimiento. Como los miembros de otros grupos, los científicos siguen un conjunto de imperativos normativos que salvaguardan su oficio y confieren sentido a su trabajo. En 1942 Merton aisló estos imperativos que se resumen en un ethos, en un complejo de valores y normas íntimamente relacionados que se consideran obligatorios para los hombres y mujeres comprometidos con las labores científicas. Estos mandatos típico-ideales incluyen: a) "universalismo" - la verdad está sometida a criterios impersonales , b) "comunismo" los productos de la ciencia son de propiedad común y toda investigación debe hacer públicos sus resultados . c) "desinterés" al científico sólo lo orienta la pasión por el saber . y d) "escepticismo organizado" —el precepto rnetodológico e institucional que reclama la cauta atención y la crítica y duda permanentes ante el legado de la ciencia. Científico que viole estas normas, camina peligrosamente por los senderos de la conducta desviada, y a poco será objeto de severas sanciones por parte de los colegas y del organismo más amplio que los representa: la comunidad científica.

Finalmente, el libro cierra sus páginas con una sugestiva coda de carácter autobiográfico: "Una vida de aprendizaje"212. Con sobriedad e ingenio Merton registra allí las tribulaciones de su infancia y adolescencia en la Philadelphia de los años diez y veinte; sus estudios universitarios en la Harvard de Pitirim Sorokin, de George Sarton y del joven Talcott Parsons; sus labores docentes en la Universidad de Columbia; su amistad con Paul Lazarsfeld; su aprendizaje con estudiantes talentosos; y los variados temas que han ocupado su atención a lo largo de los años. La conocida noción de estructura de oportunidades guía el relato y los lectores pueden observar sin dificultad cómo Merton se aprovechó de las opciones que tuvo a mano en cada etapa de su vida. Unas decisiones alcanzaron resultados positivos y otras negativos o apenas perceptibles, pero todas ellas

212 Los lectores de habla española tienen ahora la oportunidad de leerla en la Revista Colombiana de Educación, No. 33 de 1996, pp. 103-125. 
estuvieron asistidas por una férrea vocación intelectual que día tras día ha custodiado su ya casi nonagenaria experiencia vital. 